\title{
Depression and heart disease
}

\section{Editorial}

Learn about when temporary feelings of sadness are normal or when treatment is necessary to help you cope and recover. It is common for you to feel sad or depressed after a heart attack, cardiac surgery or procedure, recent hospitalization, or new diagnosis of heart disease. These emotions may be the result of not knowing what to expect or not being able to do simple tasks without becoming overly tired.

Temporary feelings of sadness are normal, and should gradually go away within a few weeks, as you get back to your normal routine and activities. Sometimes, however, a depressed mood can prevent you from leading a normal life. When a depressed mood is severe and accompanied by other symptoms that persist every day for 2 or more weeks, treatment is necessary to help you cope and recover. What is the role of depression in patients with cardiovascular disease?

Up to 15 percent of patients with cardiovascular disease and up to 20 percent of patients who have undergone coronary artery bypass graft $(\mathrm{CABG})$ surgery experience major depression. ${ }^{1}$ Studies have shown that mental stress has a negative effect on a persons heart health. In particular: Unmanaged stress can lead to high blood pressure, arterial damage, irregular heart rhythms and a weakened immune system. Patients with depression have been shown to have increased platelet reactivity, decreased heart variability and increased proinflammatory markers (such as C-reactive protein or CRP), which are all risk factors for cardiovascular disease.

For people with heart disease, depression can increase the risk of an adverse cardiac event such as a heart attack or blood clots. For people who do not have heart disease, depression can also increase the risk of a heart attack and development of coronary artery disease. In one landmark study, the continued presence of depression after recovery increased the risk of death (mortality) to 17 percent within 6 months after a heart attack (versus 3 percent mortality in heart attack patients who didn't have depression). ${ }^{2}$

During recovery from cardiac surgery, depression can intensify pain, worsen fatigue and sluggishness, or cause a person to withdraw into social isolation. Patients who have had CABG and have untreated depression after surgery also have increased morbidity and mortality. Patients with heart failure and depression have an increased risk of being readmitted to the hospital, and also have an increased mortality risk. Early research findings have indicated there may be genetic factors that increase a patients risk of depression and risk of recurrent cardiac events after a heart attack. ${ }^{3}$ Patients with heart disease and depression also perceive a poorer health status, as manifested by Quality of Life (QoL) studies. Furthermore, heart disease patients with depression have worse treadmill exercise and medication adherence than that of patients with heart disease who do not have depression. ${ }^{4-6}$

Negative lifestyle habits associated with depression such as smoking, excessive alcohol consumption, lack of exercise, poor diet and lack of social support interfere with the treatment for heart disease.

Depression has been proven to be a such a risk factor in cardiac disease that the American Heart Association (AHA) has recommended
Volume 7 Issue 6 - 2017

\section{Leo Pozuelo,' George Grant ${ }^{2}$ \\ 'Department of Psychiatry and Psychology, Canada \\ ${ }^{2}$ World organization of Natural Medicine, Canada}

Correspondence: George Grant, World organization of Natural Medicine, Richmond Hill, Canada,

Email drgrantwellness@gmail.com

Received: December 13,2017 | Published: December 18, 2017

that all cardiac patients be screened for depression using simple screening questions and an easy-to-administer survey called the Patient Health Questionnaire (PHQ-2). ${ }^{7}$ Depressive disorders result from a mix of factors. A person's family history, physical health, state of mind and environment. High levels of stress, life transitions, loss and many other factors. Imbalances in the chemicals that the body uses to control mood. How do I know when to seek help? If you are recovering from heart surgery, a heart attack, or another heart condition, temporary feelings of sadness and a depressed mood are common for the first few weeks. However, treatment is necessary when depression is severe and accompanied by other symptoms (including withdrawal from activities, not responding when visiting with family and friends, increased negative thoughts and tearfulness). Without treatment, depression can become worse. For heart patients, depression can contribute to an increased risk of heart attack and coronary disease. Talk to your health care provides who can diagnose and start depression treatment with safe antidepressants. Your health care provider also can refer you to a mental health specialist who can provide other appropriate treatment when necessary.

When depression is negatively affecting your life such as causing increased difficulties with relationships or performance at work or at home, it is important for you to get help to prevent things from getting worse. More specific reasons to seek help include: Your negative feelings, such as low mood or lack of experiencing pleasure, persist daily for 2 weeks or more. You find it increasingly difficult to participate in your recovery from heart disease. It is not uncommon for patients participating in cardiac rehabilitation to experience emotional difficulties during their physical recovery. A lack of mental drive or motivation, as well as a lack of confidence may indicate that depression has settled in. You have significant difficulty with your daily routine, social activities and/or work. You dont have anyone in whom you can confide. If you dont have anyone to share your thoughts with, its hard to know if what youre thinking makes sense. Depression also has a tendency to make people more withdrawn and isolated, making it harder to receive social support during difficult times. You have suicidal thoughts or feelings. Suicide is an irreversible solution to problems and causes permanent harm not only to yourself, but also to family members and friends. If you are having thoughts of suicide, call your physician or local 24-hour suicide hotline right away, or go to the nearest emergency room for help. 
Tips for coping with depression. Get dressed every day. Practice stress management and relaxation techniques. Get out and walk daily. Follow your prescribed exercise regimen. Ask your health care provider about a cardiac rehabilitation program. Resume hobbies and social activities you enjoy.

Share your feelings with your spouse, friend or a member of the clergy. During your recovery from surgery or a recent hospitalization, visits with friends should be limited to 15 minutes at first. Then, increase the amount of time spent with visitors, depending on how you feel.

Get a good night's sleep. Eat well-balanced, nutritious meals and follow your prescribed dietary guidelines.

Ask your health care provider about support groups that may help you cope. Support groups are available for patients who have had heart surgery and their families. Dont use harmful habits to cope, such as smoking, using drugs, drinking excessively or overeating. These harmful habits increase your risk for heart disease and stroke.

How is depression diagnosed? The biggest hurdle to diagnosing and treating depression is recognizing that someone is suffering from it. Unfortunately, approximately half of the people who experience depression are never diagnosed or treated for their illness. And not getting treatment can be life-threatening: up to 10 percent of people battling depression commit suicide.

Your health care provider can evaluate your condition by asking you to describe your symptoms. Since patients recovering from a medical illness, hospitalization or surgical procedure experience some common symptoms of depression including fatigue and insomnia, your health care provider will pay attention to these additional symptoms of depression: Withdrawal from activities Lack of reactivity from visits with family and friends Increased negative thoughts Tearfulness Sometimes, symptoms of depression can be made worse by certain medications, a physical disorder, virus or illness. Your health care provider may perform a physical exam or laboratory tests to determine if there is a physical cause for your depressive symptoms. Your health care provider will also evaluate your personal and family medical history, as well as any history of drug or alcohol use. Although there are no specific blood tests used to diagnose depression, there are various screening tools and diagnostic criteria used to make the proper diagnosis.

The U.S. Preventive Services Task Force has recommended that clinicians ask two screening questions for depression, known as the Patient Health Questionnaire (PHQ-2), including: Over the past 2 weeks, have you felt down, depressed, or hopeless? Over the past 2weeks, have you felt little interest or pleasure in doing things? If you answer yes to both of these questions, there is a high likelihood of clinical depression, and your health care provider can provide recommendations to help you get the treatment you need. Your health care provider can also administer the Patient Health Questionnaire 9 (PHQ-9), a nine-item question list that can better define your depression and guide treatment.

How is depression treated? There are many treatments for depression. Major depressive disorder may be treated with antidepressants, psychotherapy (supportive counseling or talk therapy), or a combination of both. Newer, safer antidepressant medications, such as SSRIs (selective serotonin reuptake inhibitors) have an established safety record and are safe for cardiac patients. The antidepressants sertraline (Zoloft) and citalopram (Celexa) have been the best studied, are effective and safe in patients with heart disease, and also are available in generic forms.

Psychotherapy can increase a persons social support and help the patient develop more positive thinking patterns. Brief supportive models of talk therapy appear to be the most appropriate for helping the depression that cardiac patients experience.

A healthy lifestyle including regular exercise, proper sleep, a wellbalanced diet, as well as relaxation and stress management techniques can help you manage depression. In a recent intervention trial, physical exercise was found to have a significant effect on depression. ${ }^{8}$

Major intervention studies both pharmacologic (SADHART, CREATE) and psycho-therapeutic (ENRICHD) have been performed in patients who are clinically depressed after a heart attack. These studies are helping clinicians better understand the link and treatment of depression and heart disease and guide optimal treatments. Patients with a previous history of depression or who are experiencing severe depression can be the best responders to medication intervention. ${ }^{9-13}$

\section{Conclusion}

Early detection and treatment of depression in heart patients are crucial to improve a patients quality of life and possibly prevent a recurrent coronary event. When left untreated, depression can worsen heart disease and increase the risk of a heart attack. Safe treatments are available to help you cope with depression and help you manage your heart disease.

The support and involvement of family and friends can be crucial in helping someone who is depressed. Living with a depressed person can be very difficult and stressful on family members and friends.

\section{Tips for family}

Here are some suggestions for living with a depressed person that may make things easier for you and more beneficial for the depressed person: Encourage the depressed person to seek professional help. Make sure an assessment is done by a trained mental health professional so the proper diagnosis and treatment can be initiated without delay. Educate yourself and your family about depression so you can understand what your family member is experiencing. There are many organizations and support groups that can provide more information. Know that depression is a disorder with biological, psychological, and interpersonal components; it is not a personal weakness or failure.

Help your family member follow the prescribed treatment plan and practice the coping techniques and problem-solving skills he or she is learning during psychotherapy.

Make sure medications are available if prescribed, attend therapy sessions with your family member if needed, encourage the patient to follow recommended lifestyle changes and follow up with the proper health care providers.

Recognize that depression is often expressed as hostility, rejection and irritability. Offer consistent support to your loved one. This can be draining, but it is one of the most important parts of a treatment plan. People with depression can feel alone and isolated. Giving consistent support and understanding are critical. Adopt an interaction style that puts the depressed person in charge. For example, instead 
of suggesting, Lets go to the movies tonight, try this: Id like to see a movie tonight. Which one of these do you want to see with me? Remember that treatment is effective and your loved one will start to improve in 2 to 3 weeks, achieving full improvement in a couple of months. Notice and praise any significant improvement. Reward your family member with opportunities such as visiting friends or going out for activities. Dont force these, though. Take breaks from the depressed person from time to time. It will help both of you. Consider family or marital therapy: these forms of therapy bring together all those affected by depression and help them learn effective ways to cope together.

Consider support from community support groups, either for the depressed person, or for you as the family member. 1-theanine. It comes from green tea, and its the secret behind the teas soothing, calming effects, even though its caffeinated. Studies show that 1-theanine helps you feel relaxed, yet alert totally focused, yet completely calm. ${ }^{3}$

Its like getting a massage or taking a hot bath for your mind That means, whether you need to clear your head and focus during the day, or you want to turn off your brain and drift off to sleep at night, 1-theanine has you covered. But it gets even better. Because 1-theanine also helps your brain produce serotonin, dopamine and GABA three of the most calming, soothing, feel-good chemicals in your brain. ${ }^{4}$

It even helps keep your blood pressure from riding off the rails during times of high stress protecting your blood vessels from potentially life-threatening damage. ${ }^{5}$ And Ive combined 1-theanine with. This calming cousin of the mint plant that can cut your anxiety in half Native to South-Central Europe and the Mediterranean Basin, just looking at its leaves you'd assume it was mint. But its soft lemony scent gives away its true identity. Its often used as a flavoring in teas and its essential oil is sometimes used in perfumes or aromatherapy. But in the last 10years or so, science has begun to see what natural healers have known for eons. Lemon balm is one of Natures most perfect weapons against stress and anxiety. When healthy volunteers took either lemon-balm extract or placebo, those in the lemon-balm group all reported a huge drop in their feelings of anxiety just one hour after taking it. ${ }^{6}$ And in a double-blind, placebo-controlled study, after just 15 days the group taking lemon balm reported.

Just 15 days of lemon balm helped cut anxiety nearly in half! A 49\% reduction in anxiety. A $72 \%$ reduction in anxiety-related symptoms. And a $39 \%$ decrease in insomnia. ${ }^{7}$ The symptoms of anxiety things like fatigue, feeling guilty, or unstable dropped by more than $70 \%{ }^{8}$ And, like 1-theanine, lemon balm helps calm your mind so you can focus on your day or ensure the peace and tranquility you need to drift off to sleep, quickly and easily. ${ }^{9}$

But sometimes you need more than just a calming of your mind. You need a little extra support to calm your nerves and replenish your energy to get back on track. These two legendary adaptogens know exactly what your body needs right when it needs it. In simplest terms, adaptogens help your body adapt to stress. ${ }^{10}$ Its like they magically know what you need to move past a stressful situation without losing your cool, and without feeling mentally and physically drained by it. By balancing your adrenals, hormones, and other brain chemicals, adaptogens are like natures Swiss Army knife every tool your body needs to rebalance your energy and mental focus. Especially in the face of stress.

The first is a sacred, ancient herb whose Latin name, Withania somniferous, literally means sleep inducer. Better known as ashwagandha, its stress-relieving powers rival to days most common mainstream solutions. In one study, subjects reported $44 \%$ less stress after just 60 days 12 . And in that same study people saw an average of $73 \%$ improvements in 13 . Physical feelings of stress (low energy, headaches, insomnia, low sex drive). Problems falling asleep or staying asleep. Feeling comfortable in public/social situations. Severe depression. They even saw a $28 \%$ reduction in cortisol, the stress hormone. ${ }^{14}$ Cortisol plays a vital role in your sleep cycle. But too much cortisol can leave you feeling chronically fatigued and anxious. Thats the last thing you need! Adding insult to injury, when your cortisol is chronically high it can lead to high blood sugar, high blood pressure and obesity. It can even destroy your sex drive. Ashwagandha can help ensure your cortisol levels stay right where they should be so you dont have to worry about feeling stressed (or stress making you fat!). But I have one more nutrient to tell you about. Lift your fatigue, ignite your endurance and dissolve away stress with this Arctic powerhouse The other adaptogen is called rhodiola nothing on the planet knows how to adapt to stress like this hardy little plant because it grows in some of the coldest, most brutal climates of the Arctic mountains in Northern Asia.

For centuries its been used in Russian and Scandinavian traditional medicine. Legend has it that Siberians claim it could help you live to $100 .{ }^{15}$ I cant promise youl ever see 100 candles on your birthday cake, but I do know what modern science promises. In a 10-week study, people taking rhodiola saw huge reductions in their anxiety scores. But its not just about lowering your anxiety. Ive included rhodiola because it can also help boost your endurance while lowering fatigue. It can increase your physical stamina while lowering the time it takes you to recover. You know those days where nothing goes right, you are on a deadline, you have things to get done but you are feeling mentally and physically exhausted?

All you want to do is call it quits and take a nap? Rhodiola is made for those days. No matter how many curve balls life throws at you, rhodiola makes sure you have the drive to keep going Thats why this unique combination of mental soothers, adaptogens and endurance boosters are the key ingredients in a breakthrough supplement I call, quite simply, Relax. Relax is the culmination of more than a decades research. Each ingredient carefully chosen for its targeted support to help: Soothe away your stress replace it with calm and contentment. Dissolve your feelings of anxiety at work, home, social functions, flying or traveling. Lift your feelings of dread wake up knowing that every day will be a good day. Quiet your brain chatter focus on your daily tasks or drift effortlessly to sleep Fortify your energy reserve sensuring you have the drive to tackle even your toughest day Replenish your gusto so you can take a breath, recharge and keep going Whether youre occasionally stressed out, or it follows you like a dark cloud, Relax was formulated with you in mind.

Take it daily, or when you're feeling stressed out, under the gun, or at your wits end. And because Relax works gently, with your body's natural chemistry, it's not habit-forming. And you never have to worry about negative side effects or building a tolerance to its calming effects.

\section{Acknowledgements}

None.

\section{Conflict of interest}

The author declares no conflict of interest. 


\section{References}

1. Jiang W, Davidson JR. Antidepressant therapy in patients with ischemic heart disease. Am Heart J November. 2005;150(5):871-881.

2. Frasure-Smith N, Lespérance F, Talajic M. Depression following myocardial infarction: impact on 6-month survival. JAMA. 1993;270(15):1819-1825.

3. Nakatani D, Sato H, Sakata Y, et al. Influence of serotonin transporter gene polymorphism on depressive symptoms and new cardiac events after acute myocardial infarction. Am Heart J. 2005;150(4):652-658.

4. Ruo B, Rumsfeld JS, Hlatky MA, et al. Depressive symptoms and health-related quality of life: the heart and soul study. JAMA. 2003;290(2):215-221.

5. Gehi AK, Ali S, Na B, et al. Self-reported medication adherence and cardiovascular events in patients with stable coronary heart disease: the heart and soul study. Arch Intern Med. 2005;167(16):1798-1803.

6. Gehi AK, Rumsfeld JS, Liu H, et al. Relation of self-reported angina pectoris to inducible myocardial ischemia in patients with known coronary artery disease: the heart and soul study. Am J Cardiol. 2003;92(6):705-707.

7. Litchman JH, Bigger JT Jr, Blumenthal JA, et al. Depression and coronary heart disease: recommendations for screening, referral and treatment. a science advisory from the american heart association prevention committee of the council on cardiovascular nursing, council on clinical cardiology, council on epidemiology and prevention, and interdisciplinary council on quality of care and outcomes research:endorsed by the american psychiatric association. Circulation. 2008;118(17):17681775 .
8. Blumenthal JA, Babyak MA, Doraiswamy PM, et al. Exercise and pharmacotherapy in the treatment of major depressive disorder. Psychosom Med. 2007;69(7):587-596.

9. Lesperance F, Frasure-Smith N, Koszycki D, et al. Effects of citalopram and interpersonal psychotherapy on depression in patients with coronary artery disease: The Canadian Cardiac Randomized Evaluation of Antidepressant and Psychotherapy Efficacy (CREATE) trial. JAMA. 2007;297(4):367-379.

10. Frasure-Smith N, Koszycki D, Swenson JR, et al. Design and rationale for a randomized, controlled trial of interpersonal psychotherapy and citalopram for depression in coronary artery disease (CREATE). Psychosom Med. 2006;68(1):87-93.

11. Glassman AH, Bigger JT, Gaffney M, et al. Onset of major depression associated with acute coronary syndromes:relationship of onset, major depressive disorder history, and episode severity to sertraline benefit. Arc Gen Psychiatry. 2006;63(3):283-288.

12. Glassman AH, O'Connor CM, Califf RM, et al. Sertraline treatment of major depression in patients with acute MI or unstable angina. JAMA. 2002;288(6):701-709.

13. Writing Committee for the ENRICHD Investigators. Effects of treating depression and low perceived social support on clinical events after myocardial infarction: the enhancing recovery in coronary heart disease patients (ENRICHD) randomized trial. JAMA. 2003;289(23):31063116 . 